\title{
Aberration-corrected STEM imaging and 2-D Elemental-resolved Valence-EELS Mapping of Ru-TaN Ultrathin Barrier Layer
}

\author{
Huolin L. Xin, ${ }^{*}$ and David A. Muller** \\ * Department of Physics, Cornell University, Ithaca, NY 14853 \\ ** School of Applied and Engineering Physics, Cornell University, Ithaca, NY 14853
}

In current interconnect technology for integrated circuits, a bi-layer of TaN/Ta grown by physical vapor deposition is typically used as a diffusion barrier around copper wires. This method cannot be used in the continued scaling of integrated circuits however as PVD leads to pinch-off in small geometry trenches. One potential solution is to use an atomic layer deposited (ALD) Ru-TaN mixed-phase barrier layer, which can be kept thinner while still possessing both the barrier properties of $\mathrm{TaN}$ and the direct plating character of $\mathrm{Ru}$ [1]. Developing a microscopic understanding of how the $\mathrm{Ru}$ and TaN are intermixed in the resulting thin film is critical to ensuring barrier properties are preserved as well as to improve the growth of liner materials in the continued scaling. This microscopic mixing was investigated using a $100 \mathrm{KeV} 5^{\text {th }}$-order aberration-corrected scanning transmission electron microscope (STEM) with both high spatial resolution $(\sim 1 \AA)$ and high usable current for EELS collection [2].

The $\sim 3 \mathrm{~nm}$-thick $\mathrm{Ru}-\mathrm{TaN}$ barrier layer is grown on a $\mathrm{SiO}_{2}$ substrate on a $\mathrm{Si}$ wafer using a nanolaminate approach where individual $\mathrm{Ru}$ and $\mathrm{TaN}$ layers are deposited in an alternating sequence. A plan-view TEM specimen was prepared by backside wedge polishing. Fig. 1 shows an ADF-STEM image of this specimen recorded using a $33-\mathrm{mrad} 100 \mathrm{KeV}$ aberration-corrected Nion UltraSTEM. It is found that the thin film is not uniform, yet rather is comprised of $\sim 2-3 \mathrm{~nm}$ crystalline islands separated by low density amorphous material, suggesting a phase separation of Ru and TaN. While it was very likely that these crystalline islands were Ru pockets, a 2-D elemental mapping was required to draw a confirmative conclusion. Typically, compositional analysis is done by comparing the signals from the core-loss electrons however for this sample, the total scattering cross section of N K-edge and Ta Medge are very small for a 3nm-thick thin film and the Ru M-edge overlaps with the Carbon K-edge (a typical residual in ALD growth). Rather than using the core-loss edges, the larger-cross-section valence EELS can be used for elemental mapping as well. Fig. 2 shows the low-loss reference spectra of $\mathrm{Ru}, \mathrm{TaN}$ and $\mathrm{SiO}_{2}$ respectively (normalized according to Bethe's sum rule). A spectrum recorded on the Ru-TaN layer can be decomposed into a linear combination of the three references where the resulting decomposition coefficients are proportional to the corresponding materials' concentration at the spot where the spectrum was acquired. Using this method, a 2-D distribution of Ru and TaN (Fig $3 \mathrm{a}$ and $3 \mathrm{~b}$ ) are extracted from a 64x64-pixel spectra image recorded using the UltraSTEM. To confirm this analysis, the residuals and the energy loss map at $2.2 \mathrm{eV}$ are plotted in Fig. $3 \mathrm{~d}$ and Fig $3 \mathrm{e}$ respectively. Both of them show no ordered features strongly correlated with composition maps or the simultaneously recorded ADF image indicating that the fitting is reasonable and the low-loss inelastic signal is not strongly altered by the contrast in elastic scattering.

The Ru-vs-TaN map (Fig. 3c) closely reproduces the islands' outlines in the simultaneously acquired ADF-STEM image (Fig. 3d). The Ru has islanded as expected, whereas the TaN has formed "nests" below and around the Ru. This is consistent with the ADF-STEM images: the crystalline pockets are pure $\mathrm{Ru}$ and the amorphous material is TaN. Even though atomic resolution cannot be achieved in Valence-EELS mapping due to the incident electrons' delocalized electro-magnetic field for low energy excitations [3], the 2-D composition maps show the wetting trends of $\mathrm{Ru} / \mathrm{TaN} / \mathrm{SiO}_{2}: \mathrm{Ru}$ wets to TaN more than it wets to $\mathrm{SiO}_{2}$, but it is still far from complete wetting. This result suggests why the barrier property is well preserved for such thin films -- a TaN complete wetting layer is preserved above the $\mathrm{SiO}_{2}$ preventing $\mathrm{Cu}$ from diffusing through [4]. 


\section{References}

[1] S. Kumar et al., in Interconnect Technology Conference, 2008. IITC 2008. International 2008, pp. 96.

[2] O. L. Krivanek et al., Ultramicroscopy 108, 179 (2008).

[3] D. A. Muller, and J. Silcox, Ultramicroscopy 59, 195 (1995).

[4] Research supported by Semiconductor Research Corporation and NSF MRSEC DMR\# 0520404. HLX thanks Julia A. Mundy for helpful discussions.
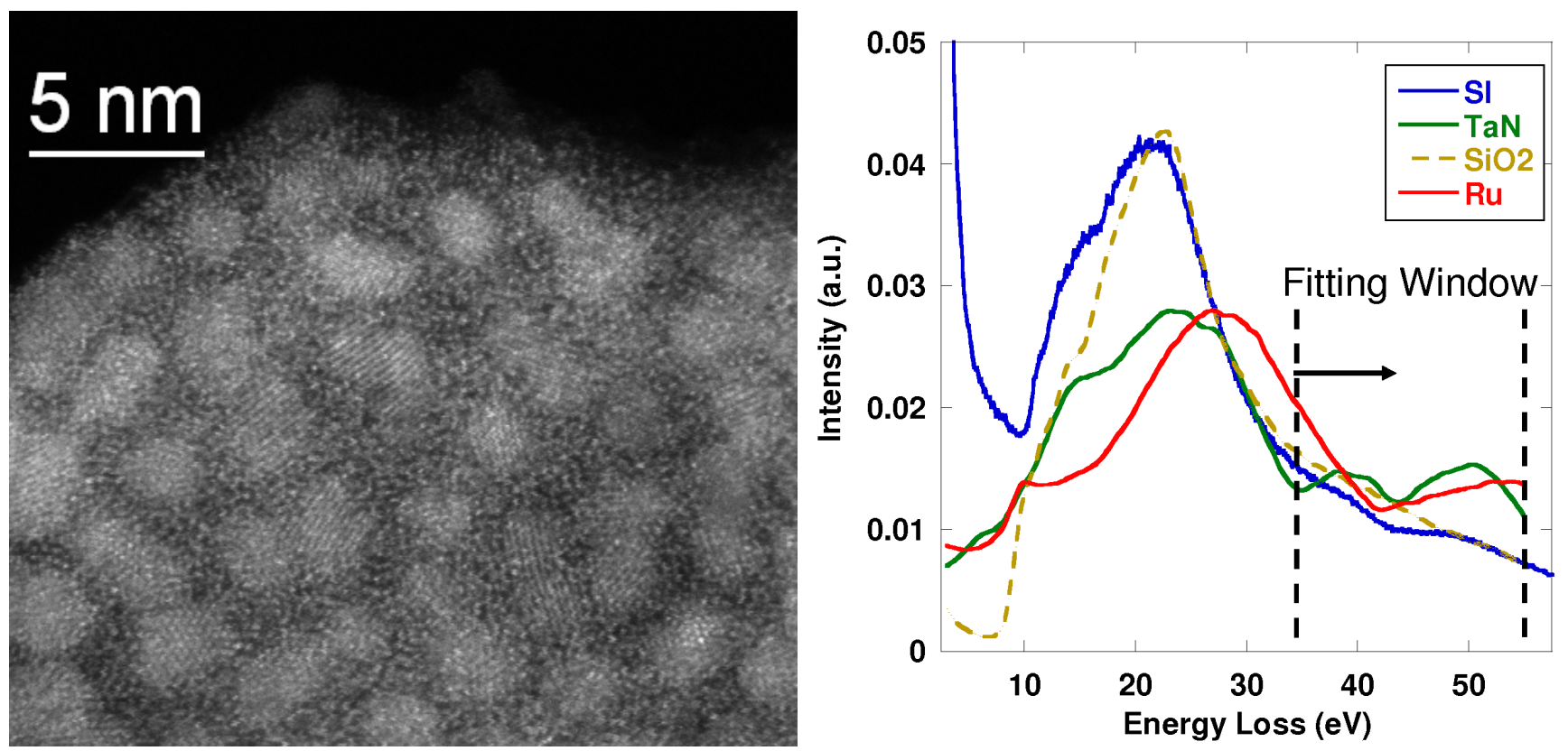

FIG. 1 (left). An ADF-STEM image of a plan-view Ru-TaN barrier layer. The lattice fringes of randomly oriented nano-islands are not detectable in uncorrected 200KeVADF-STEM images, whereas can be easily obtained in aberration-corrected ADF-STEM. It enables a quick estimation of the crystal phase and orientation of the nano-islands.

FIG. 2 (right). An averaged mapping spectrum (SI) and the $\mathrm{TaN}, \mathrm{Ru}$ and $\mathrm{SiO}_{2}$ reference spectra.
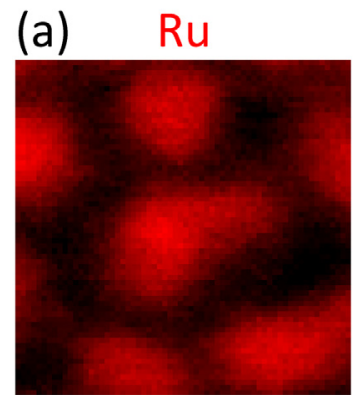

(d) Residual

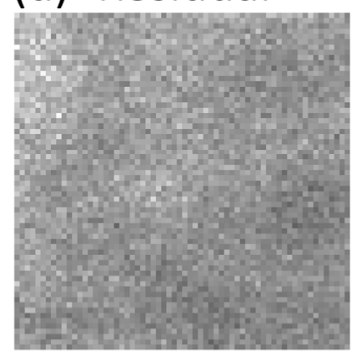

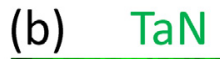

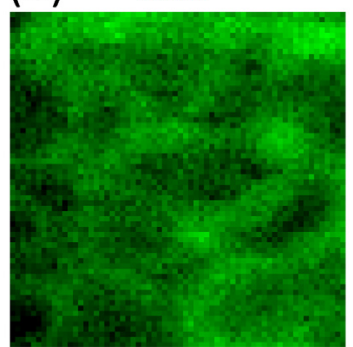

(e) $2.2 \mathrm{eV}$

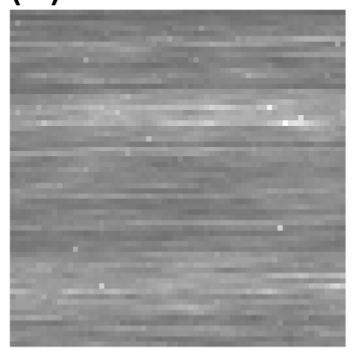

(c) Ru vs TaN

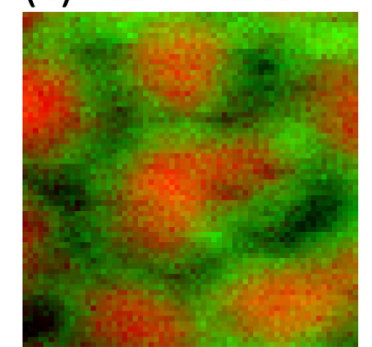

(f) ADF

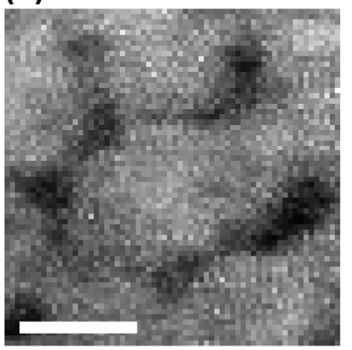

FIG. 3. 2-D Elemental-resolved mapping extracted from a $64 \times 64$-pixel spectra image by fitting features in the $42-55 \mathrm{eV}$ energy-loss window. The resulting resolution in the mapping is around $1 \mathrm{~nm}$ due to the strong delocalization in low-energy-loss inelastic scattering process. (a) the concentration distribution of $\mathrm{Ru}$; (b) the concentration distribution of $\mathrm{TaN}$; (c) the Ru vs TaN map; (d) the residual plot; (e) the electron energy loss intensity map at $2.2 \mathrm{eV}$ energy loss (the scan noise is stronger than any modulated pattern indicating the elastic scattering effect in the inelastic signal can be ignored); (f) the simultaneously recorded ADF-STEM image; the scale bar in (f) is $2 \mathrm{~nm}$ long. 